\title{
DAS DEUTSCHE IN DER KRAKAUER STADTKANZLEI IM 15. JAHRHUNDERT IM SPIEGEL DER SPRACHANALYSE AUSGEWÄHLTER WILLKÜREN
}

Das Problem der Fremdheit und des Andersseins steht in gewissem Zusammenhang auch mit der mittelalterlichen Ostsiedlung oder - besser gesagt - mit dem mittelalterlichen Landesausbau, der seine größten Ausmaße eben im Mittelalter annahm. Natürlicherweise soll man bedenken, dass die Kolonisation ein langwieriger Prozess war, der schon zur Zeit Karls des Großen (742 od. 747-814) und Ottos I. (912-973) anfing, als sie die ersten Slawen zu unterwerfen versuchten. Man darf vorläufig annehmen, dass die Anwesenheit fremder Siedler auf einem bestimmten Gebiet - und im vorliegenden Falle in Schlesien oder Kleinpolen - die Quelle der Spannungen zwischen der einheimischen und der sich neu niederlassenden Bevölkerung sein konnte, aber in dieser Beurteilung sollen wir auf keinen Fall so geradlinig sein, denn die Ostsiedlung darf nicht nur als eine aggressive Strömung des Eindringens von Deutschen in Mittel- und Osteuropa angesehen werden. In Anlehnung an Grabarek können wir die Argumente anführen, die uns erlauben, auch gute Seiten des Ostsiedlungsprozesses zu erblicken:

„Die deutschen Kolonisten brachten aber auch Fortschritt ins Land, und zwar in allen möglichen Bereichen des Lebens (Innenpolitik, Rechtswesen, Wirtschaft und Alltagsleben). Die Ostkolonisation hat also die wirtschaftliche und gesellschaftlich-kulturelle Entwicklung dieser Gebiete beschleunigt. [...] Die Bauern hofften auf Befreiung von drückenden feudalen Lasten, die Handwerker auf Freiheit in den neuen Städten sowie günstigere Produktions- und Absatzmöglichkeiten. Alle suchten nach einer gesicherten Existenz. [...] Auch für die Siedler war die Volkszugehörigkeit ihrer neuen Obrigkeit unwesentlich, sie interessierten sich in erster Linie für die ökonomischen Bedingungen, die ihnen das neue Land anbot." (Grabarek 2004, S. 511f.)

Eben diese Denkansätze lassen uns die Anwesenheit des Deutschen in seiner schlesischen Prägung in den Urkunden des Stadtrates in mittelalterlichem Krakau erklären. Die Verwendung

* Dr. Piotr Owsiński, Jagiellonen-Universität in Krakau, Institut für Germanistik, Al. Adama Mickiewicza 9a, 31-120 Kraków. E-Mail: piotr.owsinski@uj.edu.pl 
„[...] der deutschen Sprache in der damaligen Hauptstadt Polens liegt in der Expansion deutscher Siedler aus verschiedenen deutschen Sprachlandschaften östlich von Elbe und Saale. Die omdt. Kolonialmundart, die sich allmählich zur Verkehrs- und Geschäftssprache entwickelte, wurde auch in Schlesien und darüber hinaus vom deutschen Patriziat und von vielen Handwerkern deutscher Abstammung in den Städten Kleinpolens verwendet. Nach Kraków mussten nach der Verleihung des Magdeburger Rechts seit Mitte des 13. Jh. zahlreiche Bürger aus Schlesien und dem Deutschen Reich eingewandert sein und ihre Sprache fand Eingang in die bis dahin weitgehend lateinischen Aufzeichnungen der Krakauer Stadtbücher.“ (Kaleta 2004, S. 32)

Wie schon im oben angeführten Zitat angedeutet wurde, wurde Krakau am 5. Juni 1257 nach dem Magdeburger Recht gegründet. Die Stadt verdankt ihre Neugründung dem letzten kleinpolnischen Vertreter der Piasten im Mannesstamm - Boleslaus dem Schamhaften (poln. Bolestaw Wstydliwy, 1226-1279). Im herzoglichen Lokationsbrief findet sich aber die Einschränkung, dass keine Polen von Vögten aufgenommen werden dürfen. Somit gewährleistete man u. a. den deutschen Kolonisten die Rechte, sich auf einem für sie neuen Gebiet niederzulassen. In Anlehnung an Wiktorowicz (2011, S. 61ff.) beobachtet man in dieser Zeit den Zustrom der Deutschen v. a. aus Schlesien und die ständig wachsende Anzahl neuer Kolonisten führte schließlich dazu, dass man die deutsche Sprache in ihrer ostmitteldeutschen Variante immer mehr verwendete, so dass sie letztendlich auch in der städtischen Kanzlei ihre Anwendung fand. Wichtig sind auch die Mengenverhältnisse, die die Gesamtzahl der Bevölkerung Krakaus betreffen: Man schätzt, dass 10000 Einwohner im 14. Jh. in Krakau wohnten, wobei sich die Zahl der Polen auf etwa 5000 beläuft. Es gab damals 3500 Deutsche, 800 Juden, 500 Ungarn und noch etwa 200 Menschen anderer Herkunft (vgl. Mitkowski 1978, S. $31 \mathrm{ff}$.) Im 15. Jh. beobachtet man hingegen, dass sich die Zahl der Deutschen allmählich zugunsten der Polen verringerte und etwa 1/3 der Gesamtbevölkerung Krakaus betrug (Wyrozumski 1992, S. 18f.).

\section{Zum Korpus}

Die der Sprachanalyse unterzogenen Willküren wurden dem 1936 von Stanisław Ambroży Estreicher (1869-1939) in Kraków herausgegebenen Antiquum Registrum Privilegiorum Et Statutorum Civitatis Cracoviensis, dessen Schreiber bis zum heutigen Tage unbekannt sind, entnommen.

Im auf Pergament niedergeschriebenen Kodex lassen sich 4 Teile unterscheiden:

1) die Privilegien der Stadt;

2) die mit Stadtzinsen zusammenhängenden Urkunden;

3) die Willküren;

4) die Juramenta. 
Estreicher bekennt, dass der Kodex nach 1375 beginnt und nicht mit einer Hand geschrieben wurde. Das Kapitel, auf das wir unsere Aufmerksamkeit richten wollen, trägt den Titel „Dis sint willecor“ und setzt sich aus 33 Willküren aus den Jahren 1367-1387 zusammen samt vier später beigefügten Dokumenten (aus den Jahren: 1394, 1397, 1403 und 1492) (vgl. Estreicher 1936, S. IVf., XII). Im 15. Jh. wurde die Mehrheit von den Willküren durchgestrichen und mit der Randbemerkung non debet legi oder non debet versehen. Andere wurden entweder ergänzt oder geändert, was wahrscheinlich damit zusammenhängt, dass sie nicht mehr gültig im bisherigen Wortlaut waren. Es ist sicher, dass der Krakauer Stadtrat die Anfertigung des Kodexes beauftragte und ihn im 14. und 15. Jh. gebrauchte. Heutzutage befindet sich unser Kodex im Stadtarchiv Krakaus.

Nicht ohne Belang ist auch die Tatsache, dass in unserem Schriftstück - laut Estreicher, der sich wiederum auf Ambroży Grabowski beruft (vgl. Estreicher 1936, S. III) - keine Spuren vorhanden seien, die uns überzeugen könnten, dass es der städtischen Sammlung entnommen wurde. Beide behaupten, dass es eher eine zu einer Privatperson gehörende Handsammlung von Gesetzen war, die später zur Basis für die Abfassung des Codex Pictuartus von Balthasar Behem (um 1450-1508) wurde.

\section{Schreibgewohnheiten der Stadtschreiber anhand zweier ausgewählter Willküren}

Zur Grundlage der Untersuchung werden zwei folgende ausgewählte Willküren gemacht:

1) die eine ist auf 1403 datiert und hängt mit dem Schoss der unmündigen Kinder zusammen (in der Herausgabe von Estreicher: Nr. 29);

2) die andere kommt aus dem Jahre 1492 und betrifft das Wegbringen des Kots (in der Herausgabe von Estreicher: Nr. 11).

Im vorliegenden Beitrag basieren wir auf der Beziehung von Graphemen zu den wahrscheinlichen Phonemen des Mittelhochdeutschen / Frühneuhochdeutschen, z. B. kann (i) ein Allograph des mhd. Graphems $<$ ie $>$ sein. Die Grapheme kommen in spitzen Klammern vor, während ihre Varianten, die in unserem Schriftstück angetroffen werden, in runden Klammern erscheinen. Bei den Komposita werden die Positionen von Graphemen untersucht, indem man sie in bestimmten Stellungen feststellt. Daraus ergibt sich, dass sie nicht separat aufgelistet werden, so dass die Unterscheidung zwischen absolutem und gedecktem An- und Auslaut nur in den gegebenen Exempeln sichtbar ist. 
Die der Analyse unterzogenen Dokumente wurden im $15 \mathrm{Jh}$. verfasst, also sind der frühneuhochdeutschen Epoche (1350-1650) in der Periodisierung der deutschen Sprachgeschichte zuordenbar. Nach den Untersuchungsergebnissen von Wiktorowicz lassen sie sich hingegen sprachgeographisch dem ostmitteldeutschen Dialektkreis in seiner schlesischen Prägung zuzuordnen (vgl. Wiktorowicz 2011, S. 17ff., 61ff., 73ff.). Über eine solche Zuordnung entscheiden folgende sprachliche Merkmale, die von Wiktorowicz (vgl. 2011, S. 25f.) in seiner Forschung festgestellt wurden:

a) die Monophthongierung der mhd. ${ }^{1}$ Diphthonge zu langen Monophthongen [ie, uo, ye] > [i:, u:, y:];

b) die mhd. langen Vokale [i:, y:, u:] kommen noch als Monophthonge vor (also noch keine Diphthongierung);

c) die Senkung der mhd. $[\mathrm{I}, \mathrm{Y}, \mathrm{v}]>[\mathrm{e}, \propto, \mathrm{o}]$;

d) im Konsonantismus: die obd. ${ }^{2}$ Affrikate [pf] wird im Anlaut als [f] und im In- oder Auslaut als /pp/ realisiert (vgl. Wiktorowicz 2011, S. 63).

Bei den Schreibern unserer Willküren können oben genannte Spracheigenschaften erblickt werden, obwohl auch Abweichungen natürlicherweise möglich sind.

Nebensilbenvokalismus

Das tonlose [ə] wird sowohl in Gestalt des älteren schles. $<\mathrm{i}>$ als auch von $<\mathrm{e}>$ vorgefunden. Bemerkbar ist aber hier die Tendenz, dass $<\mathrm{e}>$ eher in den Vorsilben erscheint, während die Suffixe größere Schwankungen in dieser Hinsicht aufweisen:

$<$ be- $>$, $<$ ge- $>$

beslossen, bekennen, beswernisse;

geschos, vngeorbert, gesworenem, geschit, gerynne;

$<$ ver- $>$ erscheint in unserem Korpus nur als $<$ vor- $>$ : vormunde, vorschossen, vormeyden, was eins der Merkmale des Omd. ist (vgl. Wiktorowicz 2011, S. 63).

In den Suffixen finden sich hingegen beide Möglichkeiten der Wiedergabe des [ə], wobei es eher unmöglich ist, ihre Gebrauchsfrequenz festzustellen. Die Schreibung kodiert also die Nebensilbenabschwächung, obgleich auch ein paar Ausnahmen sichtbar sind (z. B. derselbin). Größere Schwankungen weist die Nebensilbe <-er> auf, wo wir auch ihre Variante (-ir) antreffen können. Beide Gestalten werden nicht konsequent benutzt und das Entstehungsdatum des Dokuments hängt damit auch nicht zusammen. Was <-et> anbelangt, so werden die nicht abgeschwächten Formen im Schriftstück aus dem Jahre 1403 vorgefunden, während

\footnotetext{
${ }^{1}$ mhd. - mittelhochdeutsch.

2 obd. - oberdeutsch.
} 
die Verben in der Willkür aus dem Ende des 15. Jh. schon die reduzierten Nebensilbenvokale enthalten (vgl. unten):

$<-\mathrm{e}>$

vormunde, eide, korcze, ringe, beswernisse, hawse, gerynne, awsrede, busse;

ansehende, truge (Konj.);

stille, reyne, sulche, ire, yre, alle, ferre, ane;

kinder, hawsir,

vnmondiger;

adi, obir;
$<-$ em $>$

gesworenem, seynem;

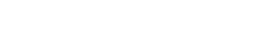

(-it) <-es $>,(-$ is $)$

(1403): gebi, firdunge, bires; hebit;

(1492): wane, doselbi, eldisti, schuttet $<$-en $>$, (-in)

herren, schosherren, greniczen;

worden, haben, beslossen, schossen, vorschossen, halden, sullen, sullens, frogen, bekennen; gesworenem, wanen, tragen; schawfelen, stossen, awsfuren, vormeyden, geben, willen, ansehende;

vnmondigen, iren;

ABER: derselbin; (!)

$<-$ ec $>$ immer als (-ik), (-ig)

wolbedochtikey, vberig;

sulche, ydirma, irkeyner;

Vokalismus:

mhd. $[\mathrm{a}]=$ schles. $<\mathrm{a}>$ :

Sowohl in den initialen als auch medialen Positionen wird ausnahmslos (a) vorgefunden:

alt, ald, also, am, vordan, allis, alle;

ratmanne, man, haben, vorbas, halden, daz, das, stat, stad, mans, hantirt, darf, wanen, wanet, tragen, ydirman;

mhd. $[\mathrm{a}:]=$ schles. $<\mathrm{a}>,(\mathrm{o})$ :

im Inlaut angetroffen; der Laut wird vorwiegend durch (a) schriftlich ausgedrückt, obwohl auch die Tendenz zu seiner Rundung und Hebung zum omd. /o:/ feststellbar ist:

ratmanne, darvmme, frogen; 
S. 85)

mhd. [a:] (nhd. [o:]) = schles. $<$ a $>($ und $<$ o $>)(v g l$. auch Kaleta 2004,

ane;

mhd. /ẹ/ und $[\varepsilon]=$ schles. $<\mathrm{e}>$ :

nur im Inlaut belegt: eyntrechticlich; wen,wenn'; hebit, derselbin, bekennen, den, greniczen, awsrede, der;

mhd. $/ \ddot{e} /=$ schles. $<$ e>, (i):

Es wurde in initialer Stellung, wo es durch (i) [is (Pron.)] vertreten wird. In medialer Stellung wird es als (e) realisiert: herren, schosherren, gelt, gebit, geben, derselbin, ansehende, vngerecht, ferre.

mhd. $[\varepsilon:]=$ schles. $<\mathrm{e}>$ :

Der Laut wurde nur einmal im Lexem: beswernisse festegestellt, wo das $<\mathrm{e}>$ im Wortinlaut steht.

Was das Graphem $<\mathrm{e}>$ noch anbelangt, so fällt auch ein anderes schlesisches Merkmal ins Auge: $<\mathrm{e}>$ als Ergebnis der Kontraktion von <-egi-, -ege->, worauf auch Kaleta (vgl. 2004, S. 90) hinweist. In unserem Korpus finden wir aber die Variante dieses Graphems in der Gestalt (ey) vor: leyt.

mhd. [I] $=$ schles. $<\mathrm{i}>$, (y):

Dieses Graphem und seine Varianten tauchen nur in der Initial- und Medialstellung auf:

ist, in (Präp.);

sint, kinder, stille, nicht, hantirt, geschit, ringe, ringk, greniczen, bis, gerynne;

Die schweren mhd. Ableitungssilben <-lich> und <-nisse> kommen als $<-$-lich $>$ und <-nisse > vor: eyntrechticlich, beswernisse;

mhd. [i:] = schles. $<\mathrm{i}>,(\mathrm{y})$ :

Mhd. [i:] wird mit $<\mathrm{i}>$ oder einmal mit (y) wiedergegeben: (Anlaut) iren, ire, yre; (Inlaut) wir.

mhd. [i:] = schles. $<\mathrm{i}>$, (y) (nach der Diphthongierung zu [ae]):

Das mhd. $<\mathrm{i}>$, das später diphthongiert wurde, wurde sowohl in der Medialstellung: vormeyden (1492); seynem (1492) als auch in der finalen Position: bey (1403), sey, sei` (1492) in beiden Schriftstücken festgestellt.

mhd. [i:] $(<$ mhd. $/$ ie $/<$ ahd. $/$ ia/, /io/) $=$ schles. $<\mathrm{i}>,(y)$ :

Es wurde im An- und Auslaut angetroffen: 
ydirman, irkeyner;

$D y / d y, w y$;

mhd. $[\mathrm{v}]=$ schles. $<\mathrm{u}>$ bzw. $(\mathrm{v})$ :

Das schles. $<\mathrm{u}>$ bzw. (v) markiert das mhd. [ひ]. Dabei wird aber auf die folgende Konsequenz gestossen: während das $<\mathrm{u}>$ im Inlaut sichtbar ist, kommt sein Alternant (v) in der Initalstellung vor:

vnd, vngeorbert, orbert, darvmme, vnlust;

jung, jungk, vnlust.

Die Verneinung < un-> erscheint immer als (vn-): vnmondigen, vnmondiger; vngeorbert; vngerecht; vnlust.

mhd. $[\mathrm{y}]=$ schles. $<\mathrm{u}>$ bzw. (o):

Das mhd. [Y] nimmt die Gestalten: (u) oder (o) in den initialen und medialen Stellungen an, wobei das Allograph (o) viel häufiger anzutreffen ist:

obir;

vorbas, vnmondigen, vnmondiger, korcze, schuttet;

Was das oben dargestellte Phänomen betrifft, so zieht unsere Aufmerksamkeit auch keine graphische Markierung des Umlauts auf sich.

mhd. [u:] = schles. $<$ aw $>$, (o):

Das Graphem samt seinen zwei Allographen wird im An- und Inlaut vorgefunden, wobei die Schreibung des Anlauts im Schriftstück aus dem Jahre 1403 keine Diphthongierung des Lautes erkennen lässt. Die Schreibung der Lexeme in der auf 1492 datierten Willkür berücksichtigt wiederum schon die lautliche Spaltung des Vokals:

awsfuren, awsrede, aws (1492);

schawfelen; hawse (1492);

Was die mhd. Präposition $u f$ anbelangt, so erscheint sie im Mitteldeutschen (Md.) in der Form nach der Kürzung und der späteren Senkung: of (1403), wo ein [o] aus dem [u:] entstanden ist (vgl. auch Kaleta 2004, S. 99).

Die Gestalt des mhd. [uo] = schles. $<\mathrm{u}>$ zeigt den schon durchgeführten Monophthongierungsprozess auf, was doch die Schreibung $<\mathrm{u}>$ im In- und Auslaut erblicken lässt:

musz, busse;

$c z u, z u$. 
mhd. [üe] = schles. $<\mathrm{u}>$ :

Die Monophthongierung des umgelauteten Zwielautes sieht man in den medialen Positionen folgender Belege: awsfuren, truge (Konj.). Die graphische Entsprechung des Lautes $<\mathrm{u}>$ zieht noch unsere Aufmerksamkeit auf die fehlende Markierung des Umlauts.

Was den mhd., später diphthongierten Vokal [y:] $(<\mathrm{iu}>)$ anbelangt, so kommt er schon als Diphthong (aw) in einem einzigen Beleg der Willkür aus dem Jahre 1492 vor: hawsirn.

mhd. [o] = schles. $<0>,(a),(u),(i)$ :

Das Graphem und seine Varianten trifft man in den initialen und medialen Positionen an.

adir, ap;

worden, beslossen, von, schossen, geschos, schosherren, vorschossen, kompt, sullen, sullens, sal, sulcher, sulche, sulch, sulchs, gesworenem, kot, vordan, schok, willen;

mhd. [o: $=$ schles. $<$ o $>$, (a):

Das lange mhd. /o:/ wird in allen drei Stellungen als $<0>$ realisiert:

ane;

gross, gros, stossen;

also, so;

mhd. $[$ ou $]=$ schles. $<$ au $>$

Dem Diphthong begegnet man nur einmal in der Initialposition: auch;

Dem mhd., in den initialen und medialen Stellungen angetroffenen Diphthong / ei / $(<$ ahd. /ei/) entsprechen vorwiegend $<$ ey $>$ und nur einmal $<$ ei $>$ in der Initial- und Medialstellung:

eyns, eyntrechticlich, eyn, eyd, ABER: eide;

irkeyner, reyne.

Das mhd. [i:], die später dem Diphthongierungsprozess unterlag, erscheint ausnahmslos in der schon diphthongierten Gestalt im In- und Auslaut als <ey>:

leyden, seyn (Pron.);

bey, sey, sei'.

Konsonantismus:

Mhd. $<$ b $>$ wird im Schlesischen lediglich als $<\mathrm{b}>$ in der Initial- sowie Medialposition und als $(\mathrm{p})$ in der finalen Stellung vorgefunden: 
vorbas, bey;

haben, gebit, geben, hebit, derselbin, vngeorbert, orbert, obir, bis, busse;

$a p$

Dem Graphem $<\mathrm{d}>$ begegnet man in allen Stellungen, wo es vorwiegend durch $<\mathrm{d}>$ oder durch ( $\mathrm{t}$ ) im Auslaut wiedergegeben wird:

$<\mathrm{d}>$ : Dy/dy, daz, das, derselbin, den, der, darf, darvmme;

worden, vnmondigen, vnmondiger, vormunde, kinder, halden, adir, vormeyden, eide, ansehende, ydirman, awsrede;

vnd, eyd, stad, vordan;

(t): sint; gelt.

Die Schreibung in der finalen Position zeugt also von der Auslautverhärtung, die doch in dieser Zeit die Wiederspiegelung in der Schrift fand.

Mhd. $<\mathrm{t}>$ kommt in allen Positionen als $<\mathrm{t}>$ oder (d) vor:

eyntrechticlich, tragen, truge (Konj.);

stat, stad, hantirt, etwas;

ratmanne, alt, ald, kompt, stat, stad, vngeorbert, orbert, leyt, hantirt, ist, nicht, vngerecht, geschit, kot, vnlust, stossen, vordan.

Einmal wird auch das Graph (tt) im Inlaut angetroffen: schuttet.

$<\mathrm{t}>$ erscheint auch in der Finalstellung als Repräsentation des /d/ (vgl. oben).

Das Graphem $<\mathrm{g}>$ kann in allen Stellungen erscheinen. In der Finalposition kann es auch durch (gk) realisiert werden, wobei ebenfalls eine Inkonsequenz bemerkbar ist:

gelt, gebit, geben, gross, gros, greniczen;

ringe, frogen, tragen, truge (Konj.);

jung, ringk;

Das mhd. /k/ wird durch (k) im An- und Auslaut realisiert:

kinder, kompt, bekennen, korcze, kot, irkeyner;

schok;

Im Auslaut kann (gk) auch ein /g/ notieren: ringk, was wieder der Ausdruck der Auslautverhärtung ist (vgl. oben).

Die Grapheme $<\mathrm{m}>$ und $<\mathrm{n}>$ erscheinen in allen Positionen. Was noch das $<\mathrm{m}>$ anbelangt, so ist auch seine Variante ( $\mathrm{mp}$ ) im Inlaut eines einzigen Lexems anzutreffen: kompt.

$<\mathrm{m}>$ : ratmanne, man, vnmondigen, vnmondiger, vormunde, mans, musz, vormeyden, ydirman;

am;

$<\mathrm{n}>$ : nicht; 
jung, jungk, vnd, sint, eyns, vnmondigen, vnmondiger, vormunde, mans, hantirt, ringe, ringk, greniczen, seynem, ane, irkeyner, reyne;

eyn, eyntrechticlich, von, wen, wenn', man, den, in (Präp.), vordan, ydirman, hawsirn;

Die Doppelzeichen $<\mathrm{mm}>$ und $<\mathrm{nn}>$ werden in der Medialposition angetroffen: ratmanne, bekennen, gerynne.

Mhd. $<1>$ taucht in allen Wortstellungen auf:

leyt, vnlust;

alt, ald, beslossen, gelt, halden, also, derselbin, sulcher, sulche, sulch, sulchs, schawfelen;

sal.

Dem mhd. $<11>$ begegnen wir lediglich im Inlaut:

sullen, sullens; stille; allis, alle; willen

Das Graphem $<\mathrm{h}>$ wird in der Initial- und Medialstellung durch (h) oder (ch) vor $<\mathrm{t}>$ realisiert:

(h): herren, schosherren, haben, halden, hebit, hantirt, hawse, hawsirn;

(ch): nicht, vngerecht.

Was noch das Graphem $<\mathrm{h}>$ anbelangt, so können wir auch sehr vorsichtig vermuten, dass es als Zeichen der Vokallänge im Wort ansehende anerkannt werden kann.

Das Digraph $<\mathrm{ch}>\mathrm{kann}$ in medialer und finaler Position vorgefunden werden: eyntrechticlich, sulcher, sulche, sulchs;

sulch;

Vor $<\mathrm{t}>$ steht (ch) auch als Variante des $<\mathrm{h}>$ : nicht, vngerecht.

Das Graphem $<3>$ kommt nur im In- und Auslaut vor, wo es durch (ss) in der medialen Position und (s), (z), (ss) und (sz) in der Finalstellung wiedergegeben wird:

(ss): beslossen, stossen, busse;

(s): vorbas, das, gros, is (Pron.), awsfuren, bis, etwas;

(z): $d a z$;

(s): gross;

(sz): musz;

Die Doppelschreibung $<33>$ ist nur in medialer Stellung als (ss) anzutreffen: schossen, vorschossen. In der Finalstellung wird $<33>$ mit (s) realisiert: geschos, schosherren. 
Das mhd. $<\mathrm{f}>$ erscheint als (f) in allen drei Wortpositionen und besitzt keine anderen Varianten, während $<\mathrm{v}>$ im Wortanlaut als (v) oder (f) und -inlaut nur als (f) zu bemerken ist:

(f): of;

(v):vorbas, von, vordan;

(f): frogen, awsfuren, ferre;

(f): schawfelen;

(f): darf;

Das Graph (v) kann auch die Repräsentation des $<\mathrm{u}>$ in der initialen Stellung sein (vgl. oben): vnd, vngeorbert, orbert, darvmme.

Dem mhd. $<\mathrm{w}>$ begegnet man in initialer und medialer Position, wo es keine anderen Alternanten hat:

worden, wen, wenn'; wanen, wanet, wy, wir, willen;

gesworenem, beswernisse, etwas;

$<\mathrm{W}>$ ist ebenfalls in der Schreibung des Diphthongs [au] sichtbar, der immer die Gestalt (aw) hat: awsfuren, awsrede, aws, schawfelen, hawse (vgl. oben).

Das Graphem < $<$ sch $>$ wird ausschließlich im Wortanlaut belegt, wo es nur durch $<$ sch $>$ realisiert wird: schossen, geschos, schosherren, vorschossen, geschit, schawfelen, schutte, schok. In der Initialstellung wird es aber als (s) festgestellt. Dies betrifft die Kombinationen /s + 1/,/s + w/ und /s + t/, was im Falle der Verbindung $/ \mathrm{s}+\mathrm{w} /$ noch nicht in der Schreibung ausgedrückt wurde, obwohl der Prozess der Entstehung des Phonems /J/ schon im 13. Jh. entstand (vgl. Moskal'skaâ 1969, S. 177; vgl. Morciniec 2015, S. 80f.):

beslossen;

gesworenem, beswernisse;

stat, stad, stille, stossen;

Die Affrikate [ts], deren graphische Entsprechung das mhd. Graphem $<\mathrm{tz}>$, bzw. $<\mathrm{z}>$ ist, wird als (cz) und einmal als $<\mathrm{z}>$ nur in der Initial- und Medialstellung angetroffen:

(cz): $c z u$;

(z): $z u$;

(cz): korcze, greniczen;

Mhd. $<\mathrm{s}>$ wird in jeder Wortposition als (s) sichtbar:

sint, so, sullen, sullens, sal, derselbin, sulcher, sulche, sulch, sulchs, ansehende, sey; 
also, ist, vnlust, seynem hawse, hawsirn;

eyns, mans;

Das mhd. Graphem $<\mathrm{r}>$, das in allen Stellungen vorkommt, besitzt auch die Variante (rr), die aber nur dreimal anzutreffen ist: herren, schosherren, ferre.

Sonst stellen wir überall $<\mathrm{r}>$ fest:

ratmanne, vngerecht, ringe, ringk, gerynne, awsrede, reyne;

worden, eyntrechticlich, frogen, iren, ire, yre, vngeorbert, orbert, hantirt, gesworenem, darf, gross, gros, korcze, vordan, tragen, truge (Konj.), greniczen, vawsfuren, beswernisse,

vorbas, derselbin, der, darvmme, wir.

Mhd. $<j>$ besitzt keine anderen Allographe und begegnet uns lediglich in der Initialposition:

jung, jungk.

\section{Schlussfolgerungen}

Den Beispielen, die oben dargestellt wurden, lässt sich eindeutig entnehmen, dass beide Willküren aus dem 15 . Jh. in der mitteldeutschen Tradition verfasst wurden, wobei sie zusätzlich schlesische Eigentümlichkeiten aufweisen:

a) die Monophthongierung [uo, ye] > [u:, y:], z. B. musz, busse, czu, zu, awsfuren, truge (Konj.);

b) die mhd. [i:, y:, u:] erscheinen schon als Diphthonge, z. B. bey, sey ,sei‘, vormeyden, seynem, hawsirn, awsfuren, awsrede, aws, schawfelen, hawse;

c) die Senkung der mhd. $[\mathrm{Y}, \mathrm{v}]>[\propto, \mathrm{\jmath}]$ wurde festgestellt: vngeorbert, orbert, kompt, obir, vorbas, vnmondigen, vnmondiger, korcze. Man findet jedoch auch die Belege vor, die diese Erscheinung nicht veranschaulichen: is (Pron.), sullen, sullens. In Anlehnung an Kaleta (vgl. 2004, S. 99) wird auch die Kürzung und Senkung des [u:] zu [०] angetroffen, wo es als schles. $<0>$ erscheint: of;

d) die md. Rundung und Hebung des [a:] zum omd. [o:]: frogen;

e) die schles. Kontraktion $<$-ege- $>\mathrm{zu}<\mathrm{e}>$ : in unserem Korpus erscheint aber die Variante des Graphems (ey), z. B. leyt;

f) $\mathrm{mhd}$. /ë/ = schles. $<\mathrm{i}>$ : is (Pron.);

g) Primärumlaut $=$ schles. $<\mathrm{e}>$ : hebit, greniczen, awsrede;

h) die Vorsilbe <ver-> erscheint ausnahmslos als $<$ vor- $>$ : vormunde, vorschossen, vormeyden. 


\section{LITERATURVERZEICHNIS}

Estreicher S. (Hrsg.) (1936), Antiquum Registrum Privilegiorum Et Statutorum Civitatis Cracoviensis. Najstarszy zbiór przywilejów i wilkierzy Miasta Krakowa, Warszawa-Kraków-LódźPoznań-Wilno-Zakopane: Polska Akademia Umiejętności.

Grabarek J. (2004), Die Ostkolonisation im westslawischen und baltischen Sprachraum bis 1350. In: Werte und Wertungen. Sprach-, Literatur- und kulturwissenschaftliche Skizzen und Stellungnahmen. Festschrift für Eugeniusz Tomiczek zum 60. Geburtstag, Wrocław: Oficyna Wydawnicza ATUT - Wrocławskie Wydawnictwo Oświatowe, S. 504-512.

Kaleta S. (2004), Graphematische Untersuchungen zum Codex Picturatus von Balthasar Behem, Kraków: Wydawnictwo Uniwersytetu Jagiellońskiego.

Mitkowski J. (1978), Nationality Problems and Patterns in Medieval Polish Towns: The Example of Cracow. In: Zeszyty Naukowe Uniwersytetu Jagiellońskiego. Prace Historyczne Nr. 39, Kraków: Wydawnictwa Uniwersytetu Jagiellońskiego, S. 31-42.

Morciniec N. (2015), Historia języka niemieckiego, Wrocław: Wydawnictwo Wyższej Szkoły Filologicznej.

Moskal`skaâ O.I. (1969), Istoriâ nemeckego âzyka, Leningrad: Izdatel`stvo Prosveŝenie.

Wiktorowicz J. (2011), Die deutsche Sprache in den Krakauer Stadtbüchern des 15. und 16. Jahrhunderts. In: Krakauer Kanzleisprache. Forschungsperspektiven und Analysemethoden, Warszawa: Zakład Graficzny Uniwersytetu Warszawskiego, S. 61-71.

Wiktorowicz J. (2011), Die graphematische Analyse der deutschen Sprache in den Krakauer Stadtbüchern des 14. Jahrhunderts. In: Krakauer Kanzleisprache. Forschungsperspektiven und Analysemethoden, Warszawa: Zakład Graficzny Uniwersytetu Warszawskiego, S. 17-32.

Wiktorowicz J. (2011), Die Krakauer Kanzleisprache im 16. Jahrhundert. In: Krakauer Kanzleisprache. Forschungsperspektiven und Analysemethoden, Warszawa: Zakład Graficzny Uniwersytetu Warszawskiego, S. 73-80.

Wyrozumski J. (1992), Dzieje Krakowa. Kraków do schyłku wieków średnich, Kraków: Wydawnictwo Literackie.

Piotr A. Owsiński

\section{THE GERMAN LANGUAGE IN THE TOWN COUNCIL OF CRACOW IN $15^{\text {TH }}$ CENTURY IN THE MIRROR OF THE LANGUAGE ANALYSIS OF THE CHOSEN CHARTERS}

(Summary)

The article hereunder presents the language analysis of the chosen German charters from $15^{\text {th }}$ century, which come from the Antiquum Registrum Privilegiorum Et Statutorum Civitatis Cracoviensis published by Stanisław Ambroży Estreicher in 1936 in Cracow.

The texts of the unknown scriveners were written in the Silesian dialect, what can be proved on the basis of the way of the notation in the law. The author introduces the results of his analysis showing the Silesian characteristic features with the appropriate examples.

Keywords: Silesian dialect, East Central German, Cracow, town council, charter 